OPEN ACCESS

Edited by:

Paolo Bonaldo,

University of Padova, Italy

Reviewed by:

Ralf J. Braun,

Universität Bayreuth, Germany

Barry Boland,

University College Cork, Ireland

*Correspondence:

Xuesong Chen

xuesong.chen@med.und.edu

Received: 30 October 2015

Accepted: 18 May 2016

Published: 08 June 2016

Citation:

Chen X, Wagener JF, Ghribi O and

Geiger JD (2016) Role of

Endolysosomes in Skeletal Muscle

Pathology Observed in a

Cholesterol-Fed Rabbit Model of

Alzheimer's Disease.

Front. Aging Neurosci. 8:129.

doi: 10.3389/fnagi.2016.00129

\section{Role of Endolysosomes in Skeletal Muscle Pathology Observed in a Cholesterol-Fed Rabbit Model of Alzheimer's Disease}

\author{
Xuesong Chen*, John F. Wagener, Othman Ghribi and Jonathan D. Geiger \\ Department of Basic Biomedical Sciences, School of Medicine and Health Sciences, University of North Dakota, \\ Grand Forks, ND, USA
}

Deficits in skeletal muscles contribute not only to the functional decline in people living with Alzheimer's disease (AD), but also to $A D$ pathogenesis. We have shown that endolysosome dysfunction plays an important role in the development of $A D$ pathological features in a cholesterol-fed rabbit model of AD. Interestingly we observed in skeletal muscle from the rabbit $A D$ model increased deposition of $A \beta$, phosphorylated tau, and ubiquitin. Here, we tested the hypothesis that endolysosome dysfunction commonly occurs in skeletal muscle and brain in this rabbit model of AD. In skeletal muscle of rabbits fed a $2 \%$ cholesterol-enriched diet for 12 weeks we observed the presence of abnormally enlarged endolysosomes, in which were increased accumulations of free cholesterol and multiple AD marker proteins subject to misfolding and aggregation including $A \beta$, phosphorylated tau, and ubiquitin. Moreover, in skeletal muscle of rabbits fed the cholesterol-enriched diet we observed decreased specific activities of three different lysosome enzymes. Our results suggest that elevated levels of plasma cholesterol can disturb endolysosome structure and function as well as promote the development of AD-like pathological features in skeletal muscle and that these organellar changes might contribute to the development of skeletal muscle deficits in $A D$.

Keywords: Alzheimer's disease, muscle, LDL, endolysosome, amyloid beta, phosphorylated tau, ubiquitin

\section{INTRODUCTION}

Alzheimer's disease (AD) is the most common neurodegenerative disorder of old age that results in massive health care costs in the United States (Hebert et al., 2013). AD is characterized clinically by a progressive cognitive impairment and pathologically by neurodegeneration and the presence of amyloid plaques composed of amyloid beta $(\mathrm{A} \beta)$ protein and neurofibrillary tangles composed of phosphorylated tau (Holtzman et al., 2011; Goate and Hardy, 2012). Besides neurodegeneration and cognitive impairment, $\mathrm{AD}$ patients often exhibit loss of muscle mass and reduced muscle strength, and such deficits in skeletal muscle may be early signs of $\mathrm{AD}$ and as such these deficits may help predict the onset and progression of clinical AD (Buchman et al., 2007; Boyle et al., 2009; Burns et al., 2010). Furthermore, skeletal muscle dysfunction can lead to progressive functional problems in $\mathrm{AD}$ patients and some have proposed that these changes may be not only concordant to those 
occurring in brain, but also that the skeletal muscle changes may serve as a potential biomarker for the onset and progression of $\mathrm{AD}$ (4-6). Although it is not known how such skeletal muscle deficits are developed in $\mathrm{AD}$, a testable hypothesis is that common pathogenic processes occur in brain as well as skeletal muscle and that these changes may help explain observations of elevated levels of $\mathrm{A} \beta$ in skeletal muscle of $\mathrm{AD}$ patients (Kuo et al., 2000).

Altered cholesterol homeostasis in general and elevated plasma LDL cholesterol in specific continues to represent robust risk factors of sporadic AD (Solomon et al., 2009; Chen et al., 2010; Lesser et al., 2011; Hui et al., 2012; Reed et al., 2014). Others and we have shown that rabbits fed a cholesterol-enriched diet exhibit pathological hallmarks of $\mathrm{AD}$ including increased levels of $\mathrm{A} \beta$, phosphorylated tau, and synaptic disruption (Sparks et al., 1994; Ghribi et al., 2006; Chen et al., 2010). Mechanistically, we demonstrated in such rabbits that elevated levels of plasma cholesterol disrupted blood-brain barrier integrity (Ghribi, 2006; Chen et al., 2008a), increased brain levels and the neuronal endolysosome accumulation of apoB (the exclusive apolipoprotein of LDL cholesterol that is normally found only in the periphery), disturbed the structure and function of neuronal endolysosomes, and led to the appearance of pathological features of $\mathrm{AD}$ including disrupted synaptic integrity, brain deposition of $\mathrm{A} \beta$, and tau pathology (Chen et al., 2010). Thus, LDL cholesterol coming from the systemic circulation might be altering neuronal endolysosome function and contributing to the pathogenesis of AD. Furthermore, using primary cultured neurons, we demonstrated that LDL cholesterol treatment increased endolysosome accumulation of cholesterol, enlarged the sizes and numbers of endolysosomes, and elevated endolysosome $\mathrm{pH}$ (Hui et al., 2012). Moreover, we demonstrated that such alterations in the structure and function of endolysosomes were directly involved in $\mathrm{A} \beta$ deposition, tau pathology, and disrupted synaptic integrity (Hui et al., 2012). Thus, our findings suggest strongly that elevated levels of LDL cholesterol contribute to pathogenesis of $\mathrm{AD}$ by disturbing the structure and function of neuronal endolysosomes.

Cholesterol for neurons is supplied by astrocyte-derived lipoproteins. Similarly, cholesterol for skeletal muscle is supplied by plasma lipoproteins (Spady and Dietschy, 1983). Extracellular cholesterol, mainly in the form of LDL particles, is delivered into muscle cells via receptor-mediated endocytosis and transported to endolysosomes (Brown and Goldstein, 1986). Importantly, we have shown that the same cholesterol-fed rabbits that develop AD-like pathology in brain (Chen et al., 2010) exhibit increased deposition of $\mathrm{A} \beta$ and phosphorylated tau in skeletal muscle fibers (Chen et al., 2008b). Because of the link between LDL cholesterol and endolysosomes and because altered endolysosome dysfunction plays an important and early role in the pathogenesis of AD (Nixon, 2005; Rajendran et al., 2008; Shimizu et al., 2008; Sannerud et al., 2011; Wolfe et al., 2013), we hypothesize that elevated levels of plasma LDL cholesterol disturb the structure and function of endolysosomes and promotes to the development of $\mathrm{AD}$-like pathological features in skeletal muscle thus contributing to the skeletal muscle deficit observed in AD. Herein, we report that cholesterol-enriched diet induced morphological and functional changes of endolysosomes as well as abnormal accumulations of ubiquitin, phosphorylated tau, and $A \beta / A \beta P P$ in skeletal muscle endolysosomes.

\section{MATERIALS AND METHODS}

\section{Rabbits}

New Zealand white female rabbits ( 1.5 to 2 years old) weighing 3 to $4 \mathrm{~kg}$ were fed either normal chow $(n=5)$ or a normal chow supplemented with $2 \%$ cholesterol $(n=9)$. After 12 weeks on the diet, animals were anesthetized and euthanized, and skeletal muscle (triceps) was dissected, frozen on liquid nitrogen cooled surface, and stored at $-80^{\circ} \mathrm{C}$ until taken for experimentation. The animal protocol was approved by the University of North Dakota Animal Care and Use Committee adherent with the Guide for the Care and Use of Laboratory Animals (NIH publication number $80-23$ ).

\section{Immunohistochemistry}

Cryostat-sectioned skeletal muscle (thickness $14 \mu \mathrm{m}$ ) was stained for target proteins using antibodies to EEA1 (Santa Cruz, sc-0415), LAMP2 (Santa Cruz, sc-8101), cathepsin D (Sigma, c0715), A $\beta$ (4G8, Signet, 39220), phosphorylated tau (SMI-31, Covance, 141815001), ubiquitin (Santa Cruz, sc-8017), N-terminal A $\beta P P$ (Chemicon, MAB348), C-terminal A $\beta P P$ (Sigma, A8717), and microtubule-associated protein light chain 3 (LC3, Santa cruz, sc-16755). For immunohistochemistry for bright field microscopy, sections were developed with diaminobenzidine substrate using the avidin-biotin horseradish peroxidase system (Vector Laboratories) and counterstained with hematoxylin, and bright field images were taken by a Nikon Eclipse 80i (upright) microscope with a $40 \times$ PlanApo objective. Double fluorescence staining was used to determine co-localization of early endosome marker (EEA1, Abcam ab70521) with late endosome/lysosome marker (LAMP2, Santa Cruz, sc-8101) or autophagosome marker (LC3, Santa cruz, sc-16755) and subcellular co-distribution patterns of $\mathrm{A} \beta \mathrm{PP}$, $\mathrm{A} \beta$, phosphorylated tau, and ubiquitin in early endosome EEA1 (Santa Cruz, sc-0415) and lysosomes (LAMP2, Santa Cruz, sc-8101) or autophagosomes (LC3, Santa cruz, sc16755). Sections were examined by an Olympus FV300 laser scanning confocal microscope: Argon laser (488 nm, $10 \mathrm{~mW}$ ) and HeNe laser $(543 \mathrm{~nm}, 1 \mathrm{~mW})$, external two-channel photomultiplier detection, FITC and Texas red probes, $60 \times$ oil PlanApo objective, Spot RT color CCD camera, and Fluoview software. Free cholesterol was stained with filipin (Sigma) and co-distribution of free cholesterol with endolysosomes was examined with a Leica DM4000B fluorescent microscope: $63 \times$ oil HCX PL Fluotar objective, DAPI and Texas red filters, Leica SCR camera, Leica Application Suite software. Images were process by Image J software or Photoshop CS5 software. Controls for specificity were used including staining muscle with an isotype-matched irrelevant antibody as a negative control, staining muscle with primary antibodies without 
fluorescence-conjugated secondary antibodies (background controls), and staining muscle with only secondary antibodies these controls allowed us to eliminate auto-fluorescence in each channel and bleed-through (crossover) between channels.

\section{Immunoblotting}

Skeletal muscle was homogenized mechanically in TPER extraction buffer (Pierce) at a ratio of 1:20 (w:v) in the presence of protease inhibitor cocktail (Sigma) and phosphatase inhibitors $(5 \mathrm{mmol} / \mathrm{l}$ sodium fluoride and $50 \mu \mathrm{mol} / \mathrm{l}$ sodium orthovanadate). The detergent-soluble fraction (supernatant) was isolated by centrifugation at $100,000 \times \mathrm{g}$ for $1 \mathrm{~h}$ at $4^{\circ} \mathrm{C}$. Protein concentration was determined by Bradford assay. Equal amounts of protein $(100 \mu \mathrm{g})$ from detergentsoluble fractions were resolved by SDS-PAGE under reducing conditions, transferred to PVDF membranes, and subjected to immunoblotting with antibodies to N-terminal A PPP (1:1000, Chemicon, MAB348), phosphorylated tau (AT8, 1: 1000, Pierce, MN1020), tau 5 (1:1000, Calbiochem 577801), acid phosphatase (1:1000, Abcam, ab54720), cathepsin D (1:1000, Sigma, C0715), cathepsin B (1:1000, Sigma, C6243), A $\beta$ (6E10, 1:500, Signet, 9320), and LC3 was detected using an antiLC3 antibody (which recognizes both LC3-I and LC3-II, 1:500, Abcam, ab58610). Blots were probed with secondary antibodies conjugated with horseradish peroxidase (HRP) for $1 \mathrm{~h}$ at room temperature, reacted with luminal reagent, exposed, visualized, and analyzed by LabWorks 4.5 software on a UVP Bioimaging System (Upland). Quantification was performed by densitometry and the results were analyzed and normalized (vs. averaged densitometric volume values of control rabbits). Glyceraldehyde 3-phosphate dehydrogenase (GAPDH, 1:5000, Abcam, ab8245) was used for loading controls.

\section{Lysosomal Enzyme Activity Measurement}

Acid phosphatase enzyme activity was determined using an Acid Phosphatase Assay kit (Sigma); a luminescence-based assay that uses 4-nitrophenyl phosphate as the substrate (Chen et al., 2010). Enzyme activities of cathepsin D and cathepsin B were determined using two separate assay kits (BioVision); fluorescence-based assays that use preferred MCAlabeled substrates for cathepsin-D and cathepsin B (Chen et al., 2010). Enzyme activities were expressed as relative opitcal density or fluorescence units (RFU) per $50 \mu \mathrm{g}$ of total protein. Specific activities of each enzyme were indicated by the ratio of enzyme activity to protein levels as determined by immunoblotting

\section{Statistical Analysis}

All data were expressed as mean and SEM. Statistical significance was determined with unpaired two-tail Student's $t$-test (Frequentist tests indicated that all data were normally distributed). $P<0.05$ was considered to be statistically significant.

\section{RESULTS}

\section{Cholesterol-Enriched Diet Increases Accumulation of Cholesterol in Endolysosomes and Disturbs Endolysosome Structure and Function}

Skeletal muscle has a very low capacity to synthesize cholesterol (Spady and Dietschy, 1983) and it meets its cholesterol needs mainly by taking up circulating lipoproteins. Extracellular cholesterol in the form of LDL is delivered into muscle cells via receptor-mediated endocytosis and transported to endolysosomes (Brown and Goldstein, 1986). In our cholesterolfed rabbits, serum levels of cholesterol were about 10-times higher than normal (Chen et al., 2008b). Because LDL cholesterol is endocytosed in skeletal muscle, we examined the extent to which cholesterol was accumulated in endolysosomes. Using double fluorescence staining techniques with EEA1 as a marker for endosomes, filipin for free cholesterol, and LAMP2 for lysosomes, we found that filipin-positive staining of free cholesterol codistributed with EEA1-positive endosomes (Figure 1A) and LAMP2-positive lysosomes (Figure 1B). None of these features were present in muscle from control rabbits. In addition, we found that EEA1-positive endosomes and LAMP2-positive lysosomes appeared to be enlarged (Figures 1A,B). To access further endolysosome morphological changes we stained for EEA1, LAMP2, and the lysosomal enzyme cathepsin D and found under light microscopy that endolysosome-positive signals (EEA1, LAMP2, and cathepsin D) were weak and diffuse in muscle from control rabbits, but endolysosomepositive staining was readily observed and appeared as large specifically stained aggregates in muscles from cholesterol-fed rabbits (Figure 1C). Furthermore, we found that the percentage of muscle fibers that contained such abnormally enlarged endolysosomes was $2.5 \pm 0.6 \%$ (\% to total muscle fibers examined). The percentage of impaired muscle fibers seems low, however, it is induced by cholesterol diet treatment for only 3 months. With longer treatment, we expect more muscle fibers will be affected, and this could have a significant impact for the pathogenesis of sporadic $\mathrm{AD}$, which take decades to develop.

It is not clear how such abnormally enlarged endolysosomes are developed; however, based on recent findings that EEA1 positive early endosomes could fuse with lysosomes under disrupted endocytic homeostasis (Falcon-Perez et al., 2005; Das and Pellett, 2011; Ramanathan et al., 2013), the observed abnormally enlarged endolysosomes in cholesterol fed rabbits could result from uncontrolled fusion of endosomes with late endolysosome/lysosomes. As such, we determined the co-localization of EEA1 with LAMP2, and we found that EEA1 is co-localized with LAMP2 in those abnormally enlarged endolysosomes in muscle fibers from cholesterol-fed rabbits (Figure 1D). These findings suggest that increased cholesterol uptake and subsequent endolysosome accumulation of cholesterol in skeletal muscle could disrupt endocytic homeostasis and lead to uncontrolled fusion of early endosomes with late endosomes/lysosomes. 
A
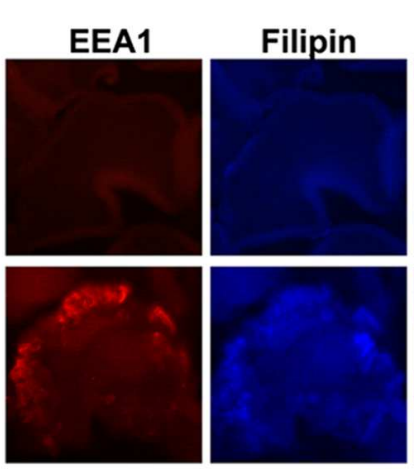

C
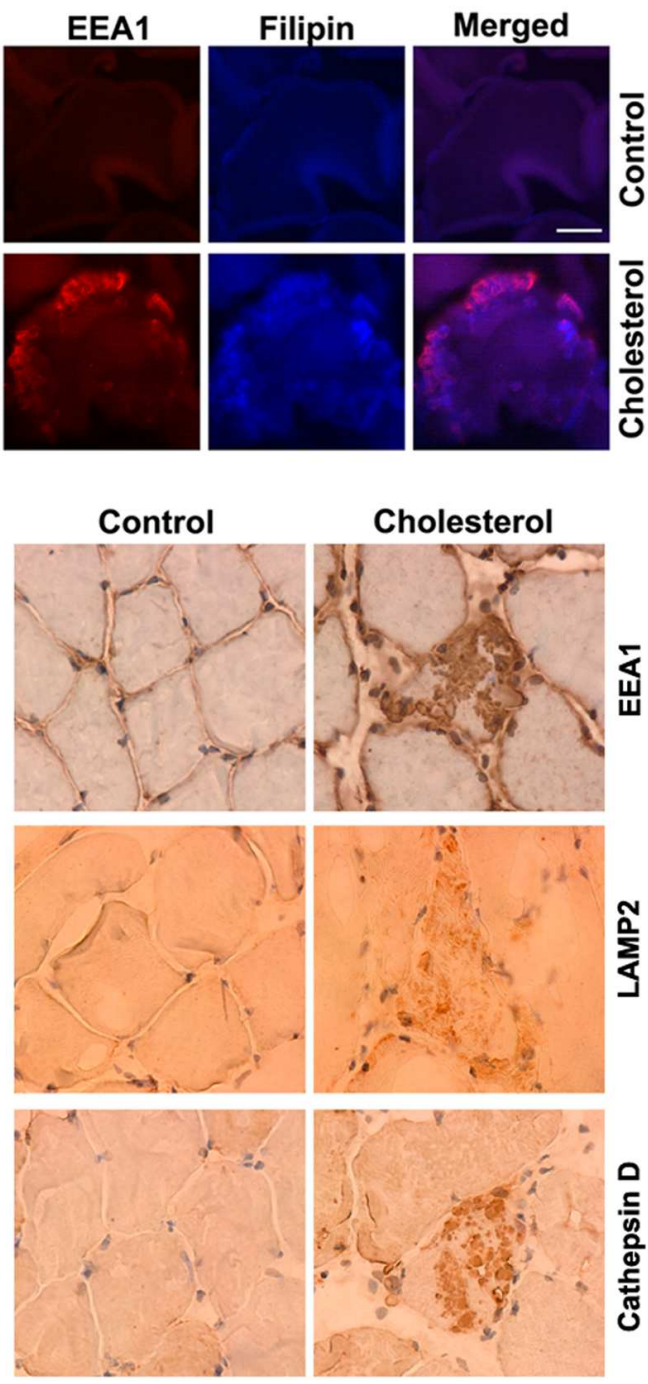

B
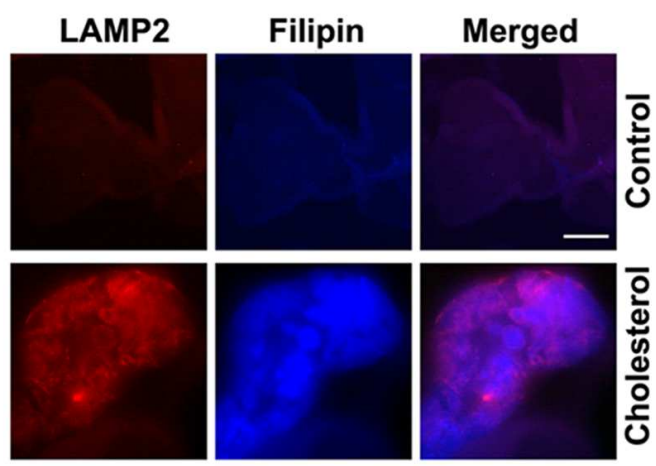

D
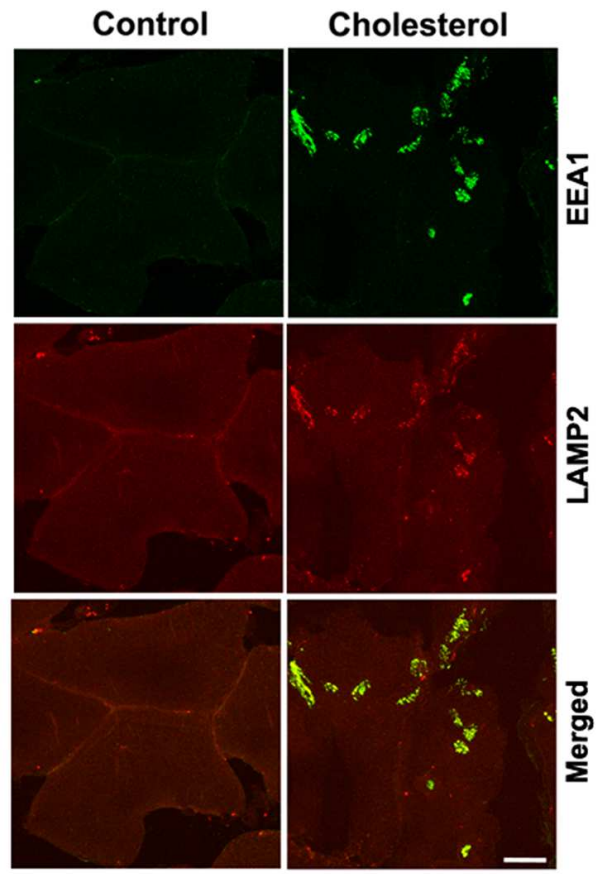

FIGURE 1 | Cholesterol-enriched diet increases accumulation of free cholesterol in endolysosomes and induces endolysosome enlargement. (A,B) Filipin-positive staining of free cholesterol (blue) co-distributed with EEA1-positive endosomes (red) and LAMP2-positive lysosomes (red) in muscle from cholesterol-fed rabbits. (C) In muscle fibers from control rabbits, EEA1, LAMP2, and cathepsin D staining appeared weak and diffuse, but in muscle fibers from cholesterol-fed rabbits, EEA1, LAMP2, and cathepsin D positive staining was strong forming large clumps (40X). (D) Cholesterol-enriched diet increased co-localization of EEA1 with LAMP2 in those abnormally enlarged endolysosomes. Bar $=20 \mu \mathrm{m}$.

Because of the above findings that cholesterol-enriched diet induced increases in cholesterol accumulation and endolysosome enlargement, we determined next the extent to which cholesterolenriched diet affected endolysosome function by measuring protein levels and specific activities of three different enzymes; cathepsin D, cathepsin B, and acid phosphatase. We found that the cholesterol-enriched diet increased significantly protein levels of acid phosphatase (Figure 2A, $p<0.05$ ), cathepsin D (Figure 2C, $p<0.05$ ), and cathepsin B (Figure 2E, $p<0.01$ ), but decreased significantly the specific enzyme activity (ratio of enzyme activity to protein levels) of acid phosphatase (Figure 2B, $p<0.05$ ), cathepsin $\mathrm{D}$ (Figure 2D, $p<0.05$ ), and cathepsin $\mathrm{B}$ (Figure 2F, $p<0.01$ ). Together, these findings indicate that the cholesterol-enriched diet increased the accumulation of cholesterol in endolysosomes and altered the structure and function of endolysosomes in skeletal muscle. As a reminder, we would like to point out that such endolysosome changes are similar to those reported by others in brain neurons from AD patients (Cataldo and Nixon, 1990; Cataldo et al., 1994, 2000) and by us in brains of cholesterol-fed rabbits (Chen et al., 2010).

\section{Cholesterol-Enriched Diet Increases the Accumulation of Phosphorylated tau, Ubiquitin, and A $\beta P P$ in Endolysosomes}

Multiple proteins that tend to aggregate including phosphorylated tau (Alonso et al., 1996; Alonso Adel et al., 2013; Wang et al., 2013), A $\beta$ (Braak and Del Tredici, 2004; 


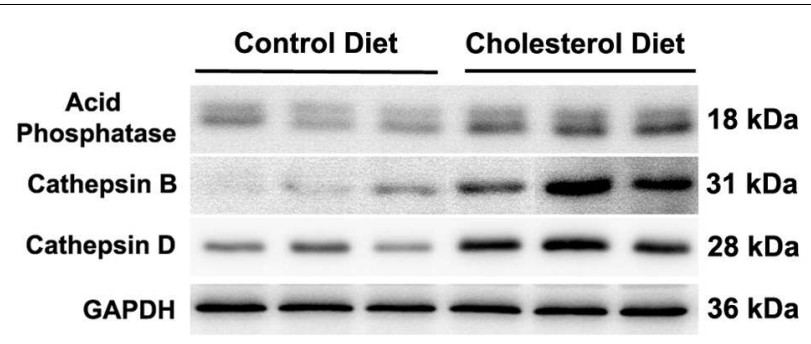

A

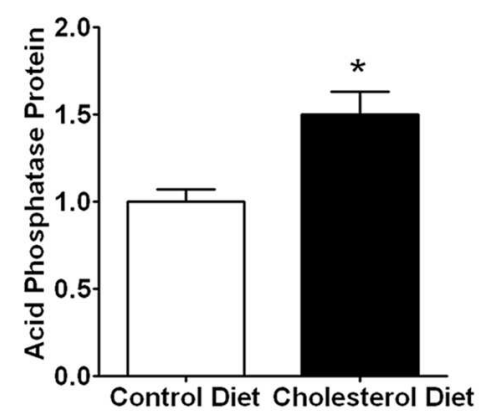

D

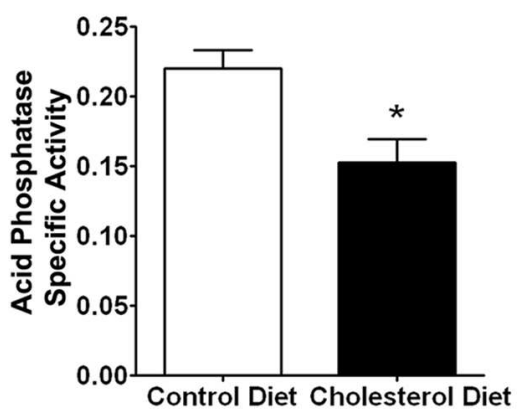

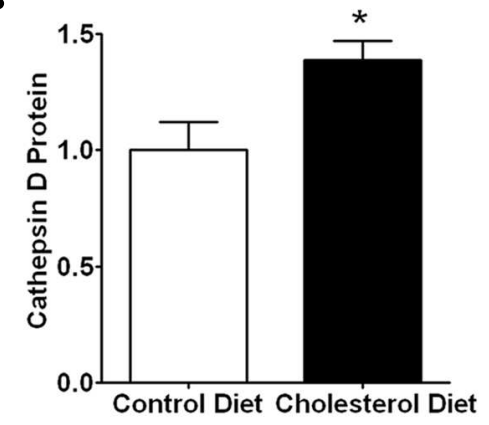

E

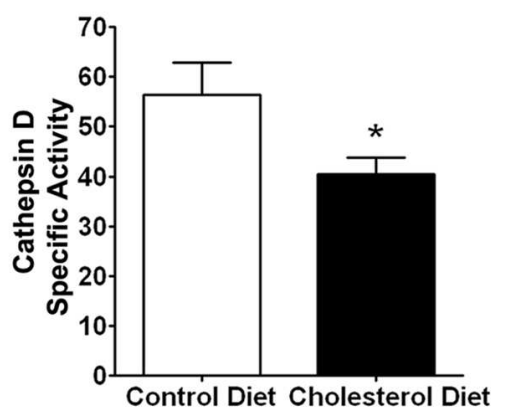

C

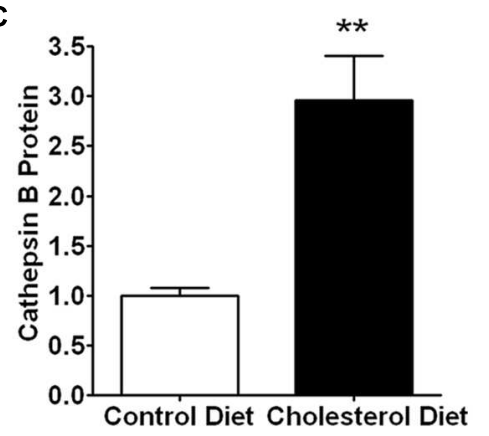

F

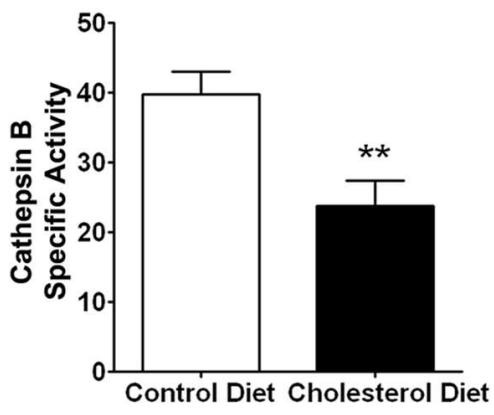

FIGURE 2 | Cholesterol-enriched diet impairs specific activities of endolysosome enzymes. Representative western blots were shown. The cholesterol-enriched diet $(n=9)$ increased significantly protein levels of acid phosphatase $\left(\mathbf{A},{ }^{*} p<0.05\right)$, cathepsin D (C, $\left.{ }^{*} p<0.05\right)$, and cathepsin B (E, $\left.{ }^{* *} p<0.01\right)$ in skeletal muscle, when compared with controls $(n=5)$. Cholesterol-enriched diet $(n=9)$ decreased significantly specific activities (ratio of total enzyme activity to protein levels as determined by immunoblotting) of acid phosphatase $\left(\mathbf{B},{ }^{*} p<0.05\right)$, cathepsin $\mathrm{D}\left(\mathbf{D},{ }^{*} p<0.05\right)$, and cathepsin $\mathbf{B}\left(\mathbf{F},{ }^{* *} p<0.01\right)$, when compared with controls $(n=5)$.

LaFerla et al., 2007), and ubiquitin (Dil Kuazi et al., 2003) are increasingly accumulated in neurons of individuals living with $\mathrm{AD}$. We have shown that these proteins are accumulated in neurons of rabbits fed a cholesterol-enriched diet (Chen et al., 2010). More importantly, we showed previously that these same proteins were also deposited in skeletal muscle of rabbits fed the cholesterol-enriched diet (Chen et al., 2008b). In addition, all these proteins have been linked to endolysosomes; amyloidogenic processing of A $\beta$ PP has been shown to occur mainly in endolysosomes following $\mathrm{A} \beta \mathrm{PP}$ endocytosis (Nixon, 2005; Rajendran and Annaert, 2012; Morel et al., 2013; Jiang et al., 2014), ubiquitin has been shown to function as a signal for membrane protein internalization and protein degradation in the autophagy-lysosome system (Holler and Dikic, 2004; D’Agostino et al., 2011), and tau and phosphorylated tau have been reported to be degraded in the autophagy-lysosome system (Kenessey et al., 1997; Oyama et al., 1998; Hamano et al., 2008; Wang et al., 2009; Chesser et al., 2013). As described above, we demonstrated that cholesterol-fed rabbits markedly enlarged endolysosomes and disturbed their function in skeletal muscle. Thus, we determined next the extent to which cholesterol-enriched diet affected accumulation of these proteins in endolysosomes in skeletal muscle of rabbits fed a cholesterol-enriched diet.

To access endolysosome accumulation of tau protein, we stained phosphorylated tau and found under bright field microscopy that SMI-31 positive phosphorylated tau signals were weak in muscle from control rabbits, but SMI-31 positive phosphorylated tau signals was readily observed and appeared as large aggregates in muscles from cholesterol-fed rabbits (Figure 3A). The size and shape of these phosphorylated taupositive signals were similar to those endolysosome-positive signals (Figure 1C), indicating the phosphorylated tau might 
A

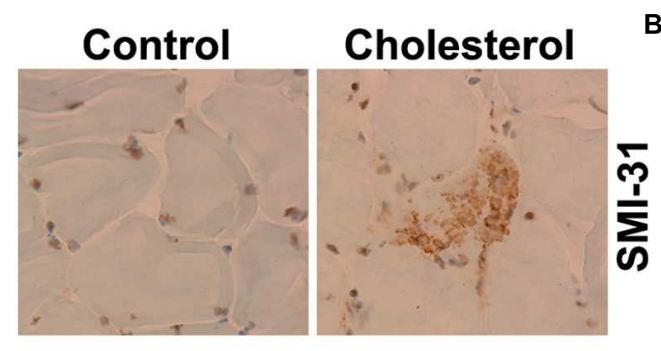

C
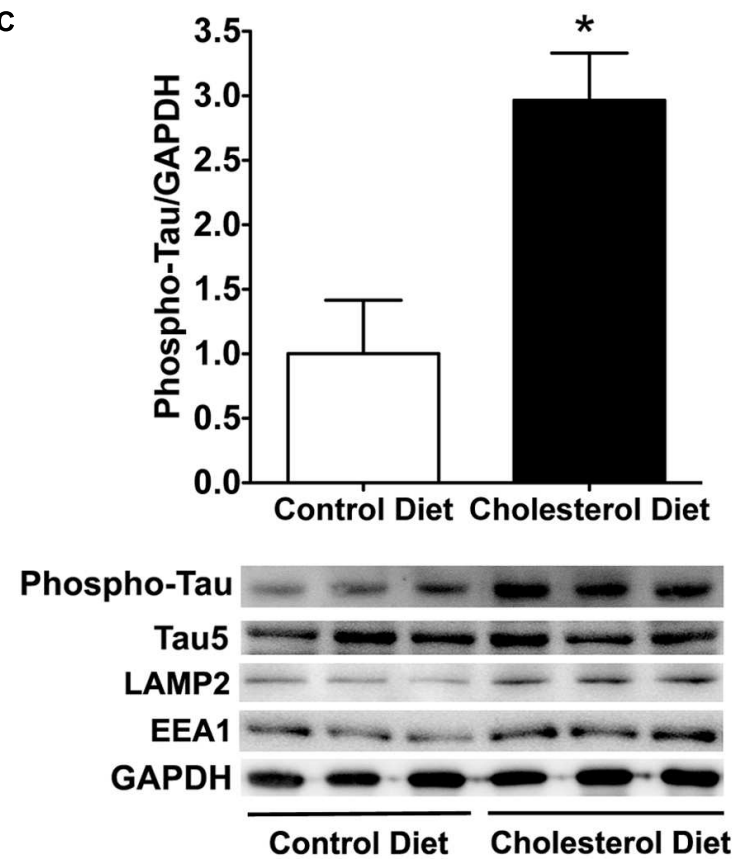

B
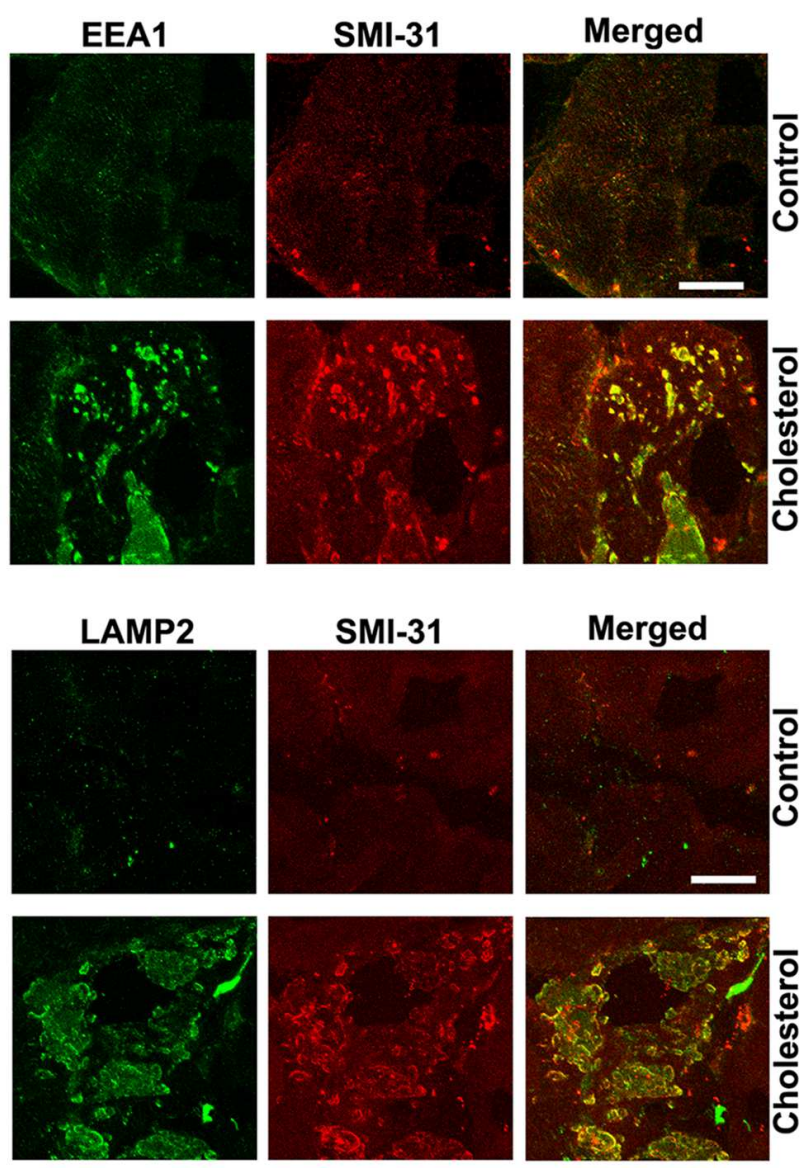

FIGURE 3 | Cholesterol-enriched diet increases the accumulation of phosphorylated tau in endolysosomes. (A) In muscle fibers from control rabbits, SMI-31 positive staining was weak, but in muscle fibers from cholesterol-fed rabbits SMI-31 positive staining appeared as large aggregates (40X). (B) SMI-31 positive staining of phosphorylated tau (red) co-distributed with EEA1-positive staining of endosomes (green) and LAMP2-positive staining of lysosomes (green) in muscle from cholesterol-fed rabbits. Bar $=20 \mu \mathrm{m}$. (C) Representative western blots were shown. Rabbits fed cholesterol-enriched diet $(n=9)$ exhibited significantly $\left({ }^{*} p<0.05\right)$ increased protein levels of phosphorylated tau in skeletal muscle, when compared with controls $(n=5)$.

be accumulating in abnormally enlarged endolysosomes. Using double fluorescence staining methods, we found that intramuscular depositions of phosphorylated tau were codistributed with EEA1-positive endosomes and LAMP2-positive lysosomes (Figure 3B). Our findings are consistent with others' reports that tau and phosphorylated tau can be degraded in the autophagy-lysosome system (Kenessey et al., 1997; Oyama et al., 1998; Hamano et al., 2008; Wang et al., 2009; Chesser et al., 2013). Although tau and phosphorylated tau are not normally present in early endosomes, under conditions when endocytic homeostasis is disrupted with uncontrolled fusion of early endosomes with late endosomes/lysosomes (Figure 1D) it is possible for tau to be co-localized with EEA1 positive early endosomes. These morphological data suggest that cholesterol-enriched diet promotes the intramuscular accumulation of phosphorylated tau in abnormally enlarged endolysosomes. To extend and confirm further our observations, we examined next protein levels of phosphorylated tau using immunoblotting methods. We found significantly increased protein levels of phosphorylated tau (Figure 3C, $p<0.05$ ) in skeletal muscle from cholesterol-fed rabbits when compared to those from control rabbits. Thus our data suggest that cholesterol-enriched diet leads to an increased accumulation of phosphorylated tau in abnormally enlarged endolysosomes in skeletal muscle.

To access endolysosome accumulation of ubiquitin, we stained ubiquitin and found under bright field microscopy that ubiquitin positive signals were weak in muscle from control rabbits, but ubiquitin positive staining was readily observed and appeared as large aggregates in muscles from cholesterol-fed rabbits (Figure 4A). The size and shape of these ubiquitin positive signals were also similar to those endolysosome-positive signals (Figure 1C), indicating the ubiquitin might be accumulating in abnormally enlarged endolysosomes. Using double fluorescence staining methods, we found that intramuscular depositions of ubiquitin were co-distributed with EEA1-positive endosomes and LAMP2-positive lysosomes (Figure 4B). 
A

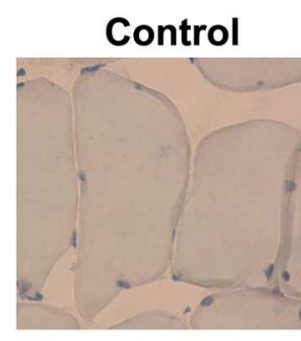

C

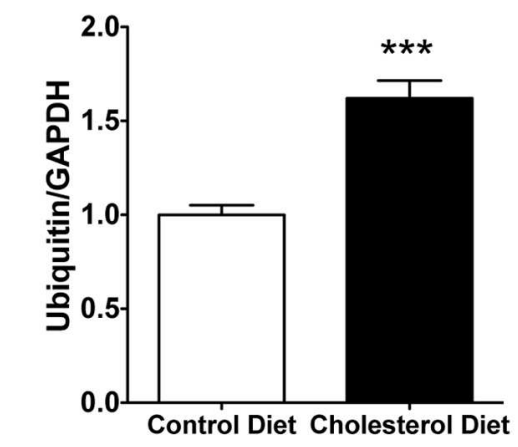

GAPDH
Cholesterol
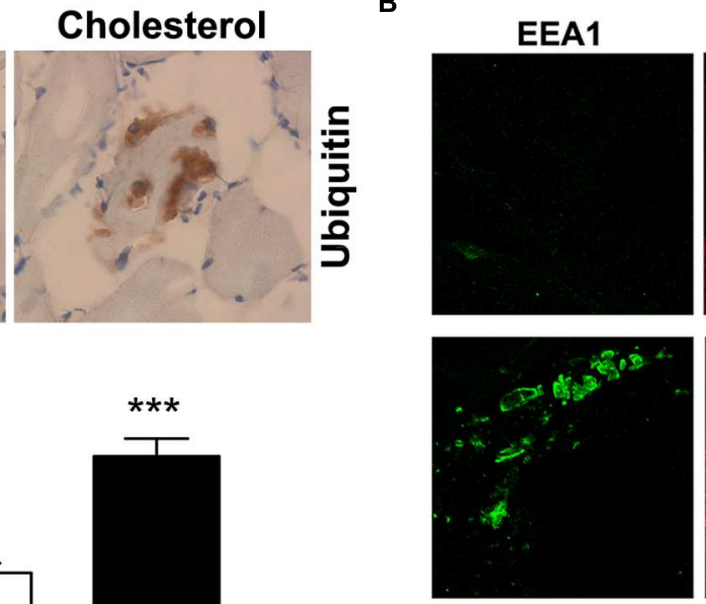

LAMP2
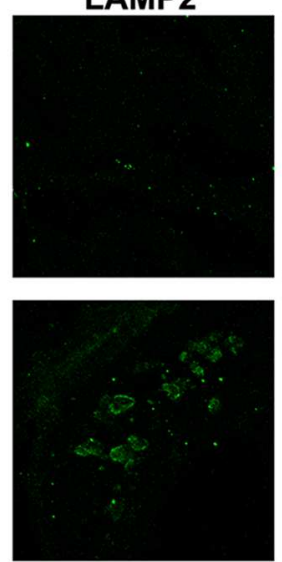

Ubiquitin
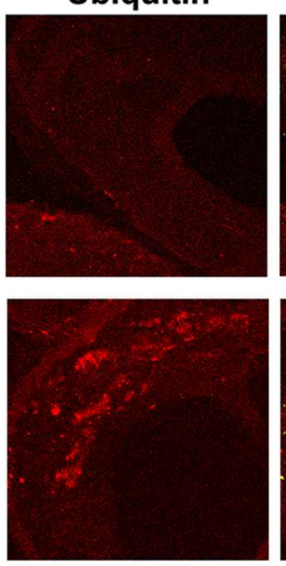

Ubiquitin
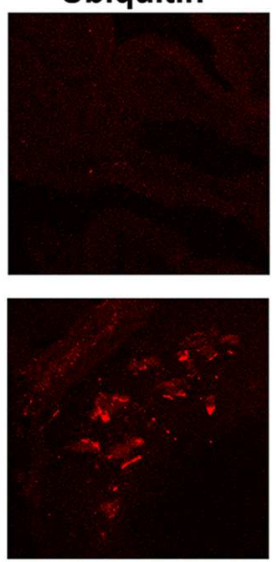

\section{Merged}
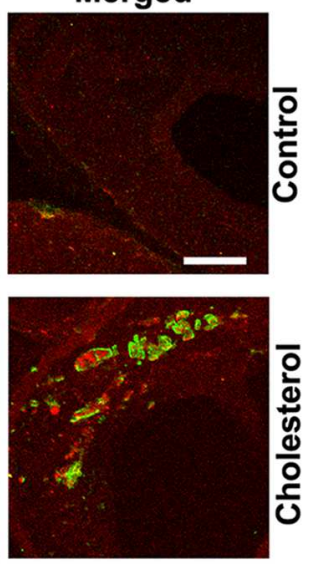

Merged
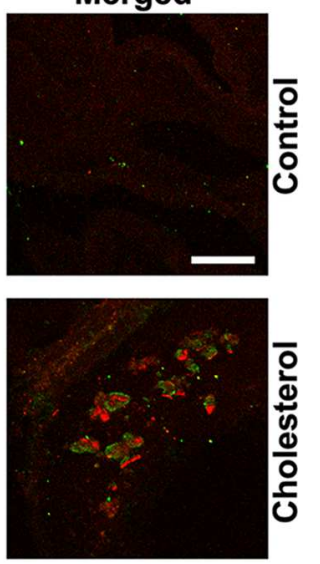

$\overline{\text { Control Diet }} \overline{\text { Cholesterol Diet }}$

FIGURE 4 | Cholesterol-enriched diet increases the accumulation of ubiquitin in endolysosomes. (A) In muscle fibers from control rabbits, ubiquitin positive staining was weak, but in muscle fibers from cholesterol-fed rabbits ubiquitin positive staining appeared as large aggregates (40X). (B) Ubiquitin positive staining (red) co-distributed with EEA1-positive staining of endosomes (green) and with LAMP2-positive staining of lysosomes (green) in muscle from cholesterol-fed rabbits. Bar $=20 \mu \mathrm{m}$. (C) Representative western blots were shown. Rabbits fed cholesterol-enriched diet $(n=9)$ exhibited significantly $(* * * P<0.001)$ increased protein levels of ubiquitin in skeletal muscle, when compared with controls $(n=5)$.

These morphological data suggest that ubiquitin accumulates intramuscularly in abnormally enlarged endolysosomes, which are consistent with others' reports that ubiquitin functions as a signal for membrane protein internalization and protein degradation in the autophagy-lysosome system (Holler and Dikic, 2004; D’Agostino et al., 2011). To extend and confirm further our observations, we examined next protein levels of ubiquitin using immunoblotting methods. We found significantly increased protein levels of ubiquitin (Figure 4C, $p<0.001$ ) in skeletal muscle from cholesterol-fed rabbits when compared to those from control rabbits. Thus, our data suggest that cholesterol-enriched diet leads to an increased accumulation of ubiquitin in abnormally enlarged endolysosomes in skeletal muscle. The presence of ubiquitin positive inclusions indicate a general degeneration process is occurring. To assess whether such general degeneration might be involved in the pathogenesis of Parkinson's disease or amyotrophic lateral sclerosis, we determined the expression of $\alpha$-synuclein and TPD-43 using immunohistochemistry. We found positive but weak immunopositive staining for $\alpha$-synuclein and TPD-43 in impaired muscle fibers (Supplementary Data). These findings are consistent with our findings that cholesterol-enriched diet induces a general degeneration process as evidenced by the presence of ubiquitin positive inclusions. However, given the weak staining signals for $\alpha$-synuclein and TPD-43, it is not likely that cholesterol-enriched diet plays a significant or specific pathological role in the development of Parkinson's disease or amyotrophic lateral sclerosis.

To access endolysosome accumulation of $A \beta P P$ protein, we stained $\mathrm{A} \beta \mathrm{PP}$ with a $\mathrm{N}$-terminal $\mathrm{A} \beta \mathrm{PP}$ antibody or a $\mathrm{C}$-terminal $A \beta P P$ antibody (data not shown) and found under bright field microscopy that A $\beta P P$ positive signals were mainly present 
at plasma membrane and intramuscular signals were weak in muscle from control rabbits, but $\mathrm{A} \beta \mathrm{PP}$ positive signals were readily observed and appeared as large aggregates inside muscles from cholesterol-fed rabbits (Figure 5A). The size and shape of these $\mathrm{A} \beta \mathrm{PP}$ positive signals were also similar to those endolysosome-positive signals (Figure 1C), indicating the A $\beta P P$ might be accumulating in abnormally enlarged endolysosomes. Using double fluorescence staining methods, we found that intramuscular depositions of $\mathrm{A} \beta \mathrm{PP}$ were co-distributed with EEA1-positive endosomes and LAMP2positive lysosomes (Figure 5B). These morphological data suggest that $\mathrm{A} \beta \mathrm{PP}$ accumulates intramuscularly in abnormally enlarged endolysosomes. To extend and confirm further our observations, we examined next protein levels of $\mathrm{A} \beta \mathrm{PP}$ using immunoblotting methods. We found significantly increased protein levels of $\mathrm{A} \beta \mathrm{PP}$ (Figure $5 \mathrm{C}, p<0.01$ ) in skeletal muscle from cholesterol-fed rabbits when compared to those from control rabbits. Thus, our data suggest that cholesterol-enriched diet leads to an increased accumulation of $\mathrm{A} \beta \mathrm{PP}$ in abnormally enlarged endolysosomes in skeletal muscle.

\section{A $\beta$ Accumulates in Endosomes and Autophagosomes in Skeletal Muscle of Rabbits Fed Cholesterol-Enriched Diet}

Endolysosomes play a critical role in amyloidogenic processing of A $\beta$ PP (Rajendran and Annaert, 2012; Morel et al., 2013; Jiang et al., 2014) and they are major sites where $A \beta$ is generated following internalization of A $\beta$ PP (Nixon, 2005; Rajendran et al., 2008; Shimizu et al., 2008; Sannerud et al., 2011; Rajendran and Annaert, 2012; Morel et al., 2013; Jiang et al., 2014). Once generated, $A \beta$ can be degraded by cathepsins in lysosomes (Miners et al., 2011) and the remaining $A \beta$ can either accumulate in endolysosomes where it may precipitate $\mathrm{AD}$ pathogenesis (Braak and Del Tredici, 2004; LaFerla et al., 2007; Zou et al., 2015) or it can be released via exocytosis (Annunziata et al., 2013; Nilsson et al., 2013). Accordingly, A $\beta$ levels can be enhanced by those factors that promote amyloidogenic processing of $\mathrm{A} \beta \mathrm{PP}$ (Grbovic et al., 2003; Ma et al., 2009) and those that impair A $\beta$ degradation (Torres et al., 2012).

Given our findings that cholesterol enriched diet increases cholesterol accumulation in endolysosomes and dramatically
A

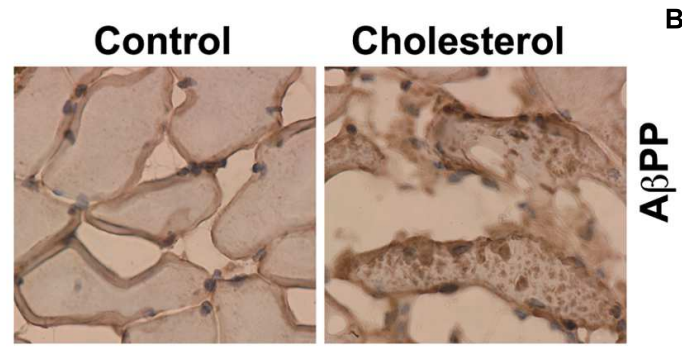

C

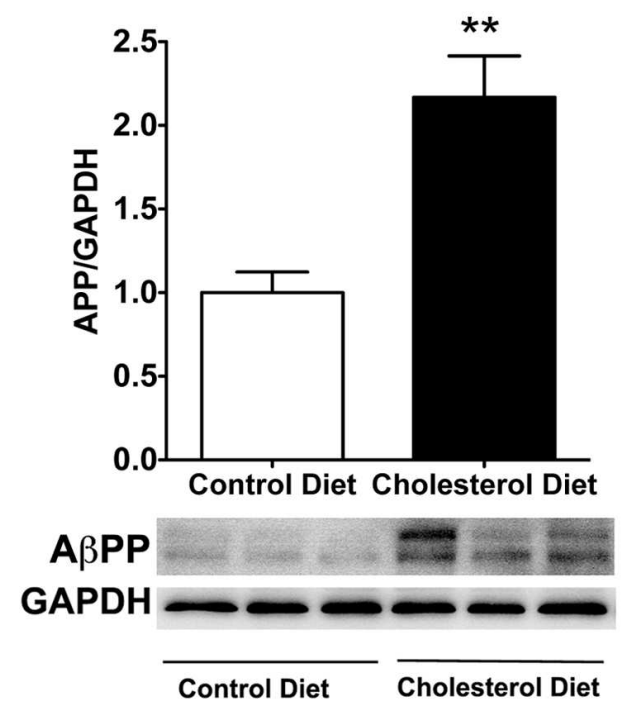

B
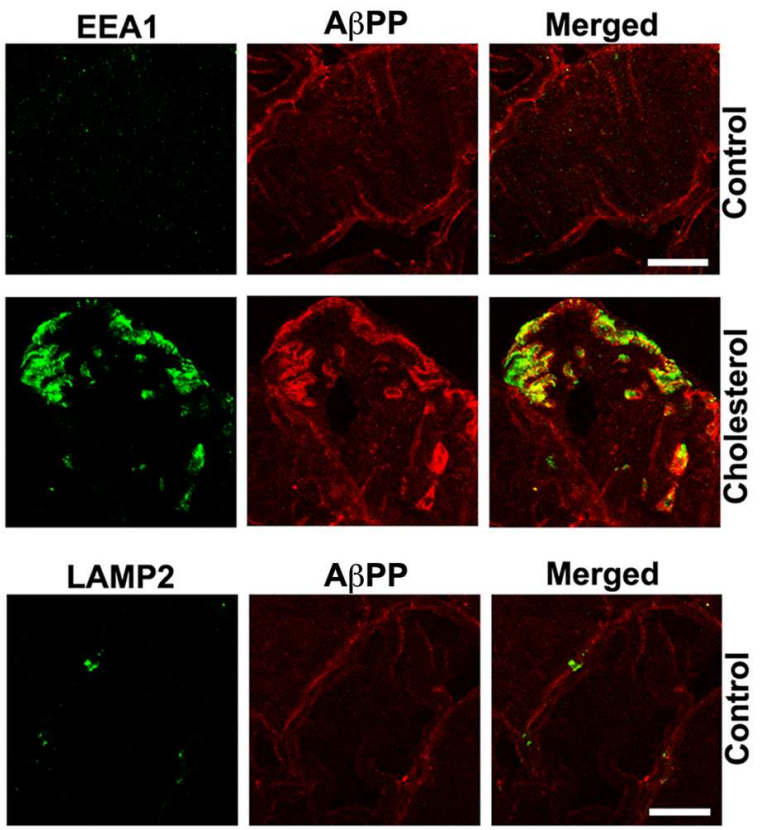

Merged
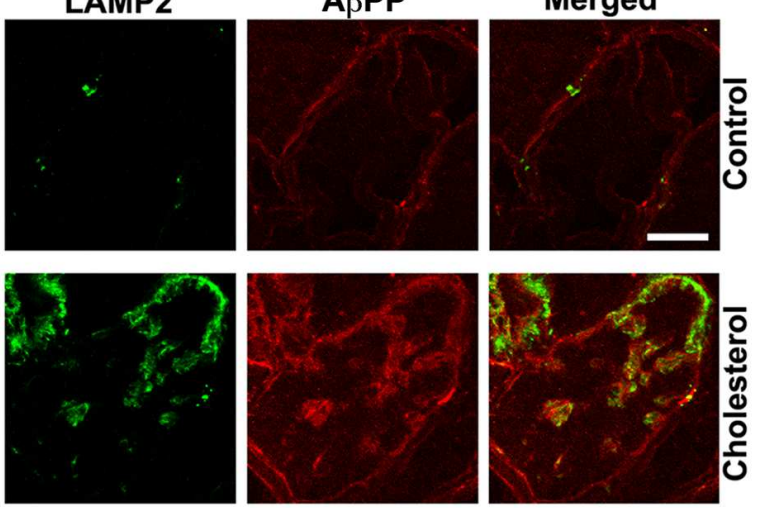

FIGURE 5 | Cholesterol-enriched diet increases the accumulation of A $\beta P P$ in endolysosomes. (A) In muscle fibers from control rabbits, N-terminal A $\beta P P$ positive signals were mainly present at plasma membrane and intramuscular signals were weak, but in muscles from cholesterol-fed rabbits A $\beta P P$ positive signals appeared as large aggregates inside muscles (40X). (B) N-terminal AßPP positive staining (red) co-distributed with EEA1-positive staining of endosomes (green) and with LAMP2-positive staining of lysosomes (green) in muscle from cholesterol-fed rabbits. Bar $=20 \mu \mathrm{m}$. (C) Representative western blots were shown. Rabbits fed cholesterol-enriched diet $(n=9)$ exhibited significantly $(* * P<0.01)$ increased protein levels of A $\beta$ PP in skeletal muscle, when compared with controls $(n=5)$. 
A

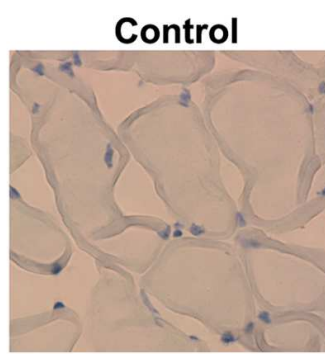

C

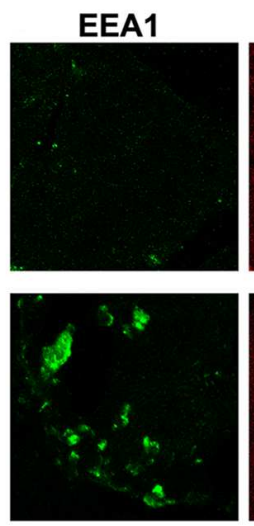

D

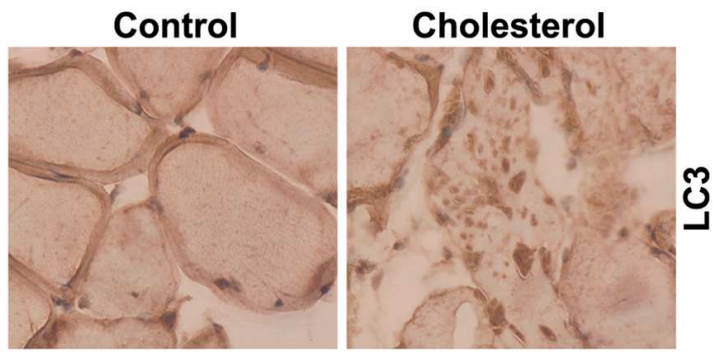

F
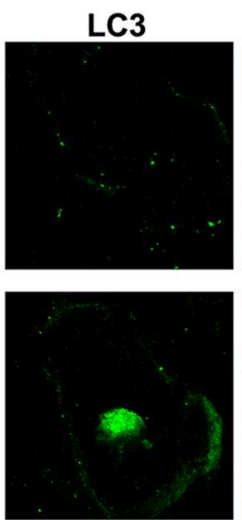

Cholesterol

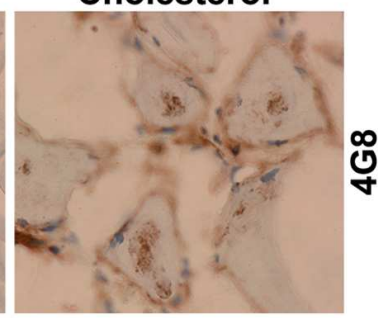

4G8
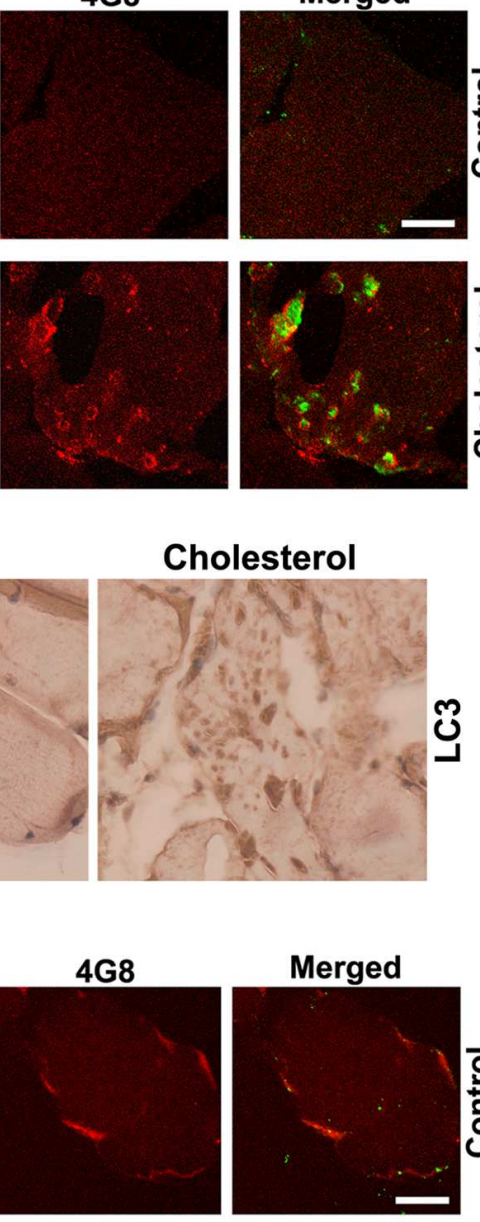
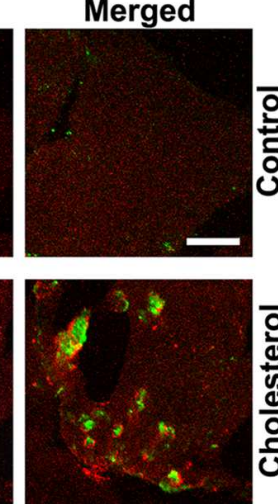

E
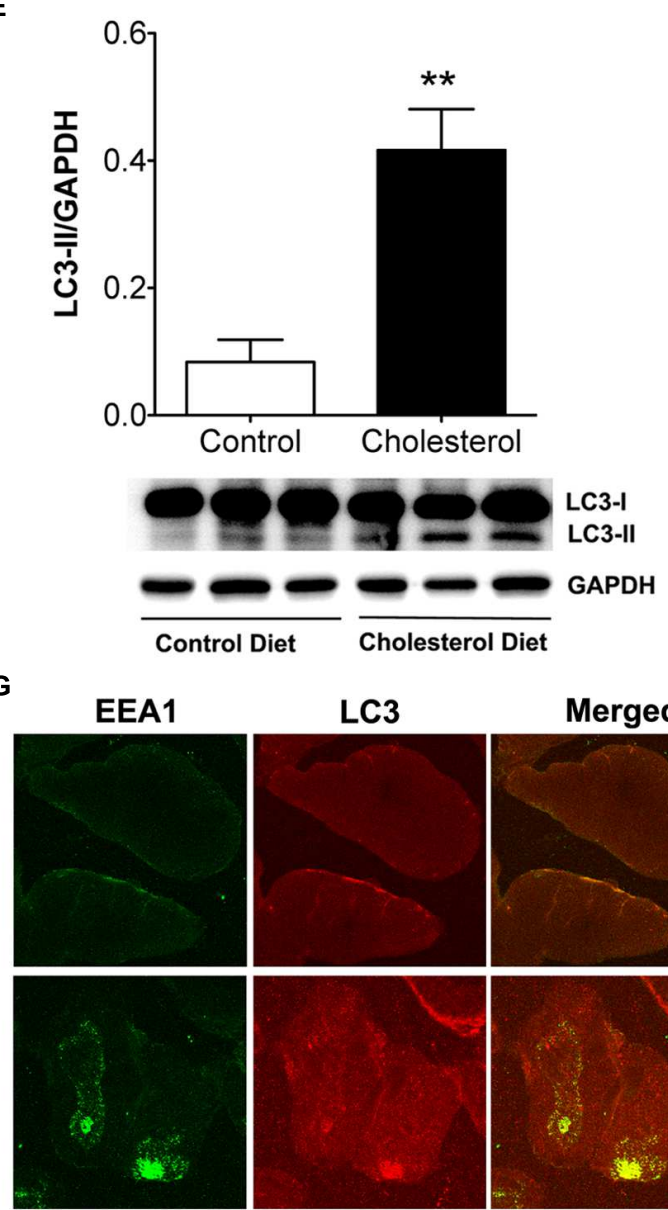

B
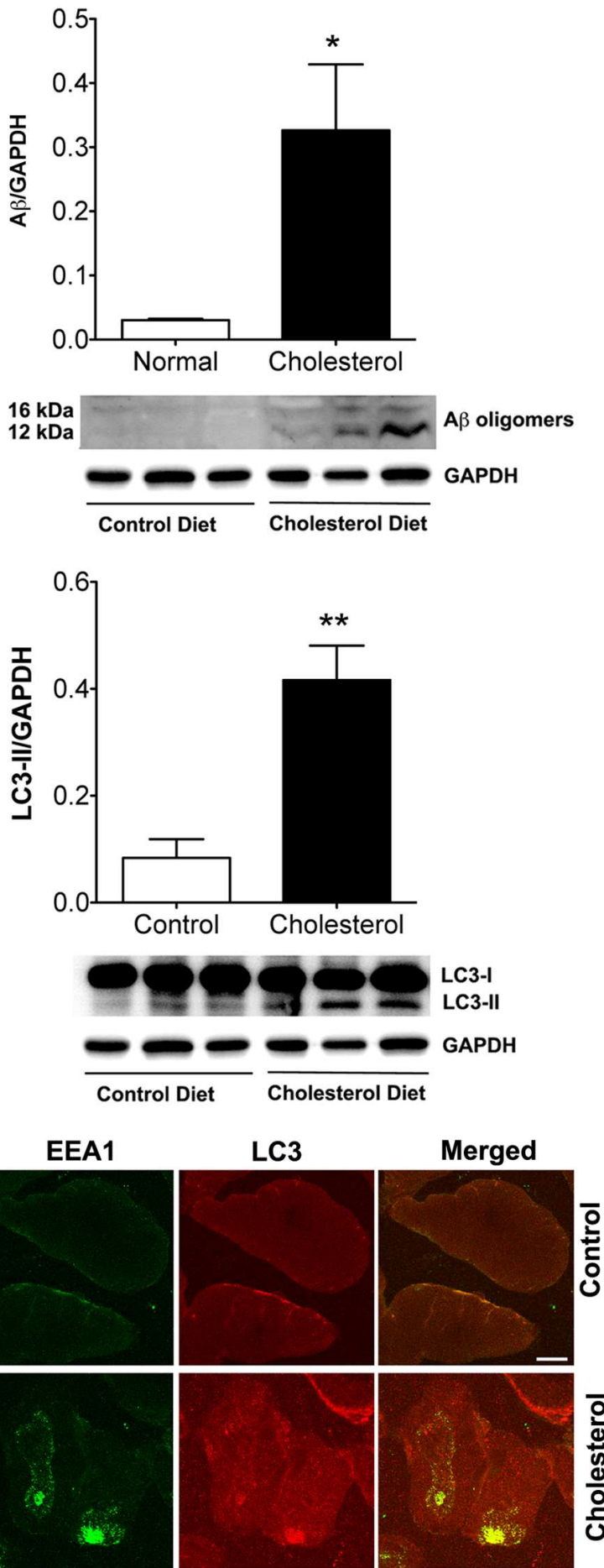

FIGURE 6 | Cholesterol-enriched diet increases deposition of $\mathbf{A} \boldsymbol{\beta}$ in endosomes and autophagosomes. (A) In muscle fibers from control rabbits, $4 \mathrm{G} 8$ positive A $\beta$ signals were weak, but in muscles from cholesterol-fed rabbits $4 \mathrm{G} 8$ positive A $\beta$ signals appeared as large aggregates inside muscles (40X).

(B) Representative western blots were shown. Cholesterol-enriched diet $(n=9)$ increased significantly protein levels of $A \beta$ oligomers $\left({ }^{*} p<0.05\right)$ in skeletal muscle, when compared with controls $(n=5)$. (C) 4G8-positive staining of A $\beta$ (red) co-distributed with EEA1 positive staining of endosomes (green) in skeletal muscle from cholesterol-fed rabbits. Bar $=20 \mu \mathrm{m}$. (D) In muscle fibers from control rabbits, LC3 positive autophagosome signals were weak and diffuse, but appeared as large aggregates inside muscles from cholesterol-fed rabbits (40X). (E) Representative western blots were shown. Cholesterol-enriched diet ( $n=9$ ) increased significantly protein levels of LC3-II (** $p<0.01)$ in skeletal muscle, when compared with controls $(n=5)$. (F) 4G8-positive staining of A $($ red) co-distributed with LC3-positive staining for autophagosomes (green) in skeletal muscle from cholesterol-fed rabbits. Bar $=20 \mu \mathrm{m}$. (G) EEA1positive endosomes (green) co-distributed with LC3-positive staining for autophagosomes (red) in skeletal muscle from cholesterol-fed rabbits. Bar $=20 \mu \mathrm{m}$. 
altered structure and function of endolysosomes, we determined the extent to which cholesterol-enriched diet affects levels of intramuscular $A \beta$. Using the $4 \mathrm{G} 8$ antibody for detection of $A \beta$, we found under bright field microscopy that $4 \mathrm{G} 8$ positive $A \beta$ signals appeared as large aggregates inside muscles from cholesterol-fed rabbits (Figure 6A). Using immunoblotting methods, we found that cholesterol-enriched diet increased significantly protein levels of $A \beta$ oligomers (Figure 6B, $p<0.05$ ) in skeletal muscle from cholesterolfed rabbits. Using double fluorescent staining, we found that 4G8-positive $\mathrm{A} \beta$ staining co-distributed with EEA1positive endosomes (Figure 6C), a finding consistent with endosome production of $\mathrm{A} \beta$. None of these features was present in skeletal muscle of control rabbits. Our observations of increased accumulation of $\mathrm{A} \beta / \mathrm{A} \beta \mathrm{PP}$ in endolysosomes suggests to us that increased amyloidogenic processing of $A \beta P P$ was caused by morphological and functional alterations in endolysosomes.

Because $A \beta$ was also found to be a substrate for autophagy (Nixon, 2007), we next determined the extent to which $\mathrm{A} \beta$ accumulated in autophagosomes. We found under light microscopy that LC3 positive autophagosome signals were weak and diffuse in muscles from control rabbits, but appeared as large aggregates inside muscles from cholesterolfed rabbits (Figure 6D). Using immunoblotting methods, we found that cholesterol-enriched diet increased significantly protein levels of LC3-II, an indicator of autophagosome proliferation and maturation (Figure 6E, $p<0.01$ ). Using double fluorescent staining, we found that 4G8-positive $\mathrm{A} \beta$ staining co-distributed with LC3 positive autophagosomes (Figure 6F). The observation that $A \beta$ is present in LC3-positive autophagosomes suggests further the presence of dysfunctional endolysosome-autophagosome system. Given our finding that cholesterol-enriched diet increases the co-localization of EEA1 with LAMP2 (Figure 1D), which indicates endolysosome accumulation of cholesterol could lead to uncontrolled fusion of early endosomes with late endosome/lysosomes, we determined the extent to which cholesterol-enriched diet affects the colocalization of EEA1 with LC3. We found that cholesterolenriched diet increased dramatically co-localization of EEA1 with LC3 (Figure 6G). These observations indicate that endolysosome accumulation of cholesterol could also increase fusion of early endosomes with autophagosome, a phenomenon that fosters maturation of autophagosomes (Tooze and Razi, 2009).

\section{DISCUSSION}

The present studies were aimed to test the hypothesis that endolysosome dysfunction in skeletal muscle shares common pathological features to those found in brain in a rabbit model of sporadic AD. Principally, we demonstrated that skeletal muscle from a cholesterol-fed rabbit model of $\mathrm{AD}$ exhibited increased cholesterol accumulation in endolysosomes, enlarged endolysosomes, and impaired endolysosome function. Furthermore, we demonstrated that various $\mathrm{AD}$ marker proteins including phosphorylated tau, ubiquitin, $A \beta P P$ and $A \beta$ were accumulated in those enlarged endolysosomes. Together these findings provide further insight into skeletal muscle involvement in $\mathrm{AD}$ and the potentially significant role that endolysosomes play in the pathogenesis of $\mathrm{AD}$.

Endolysosomes are acidic organelles consisting of endosomes, lysosomes, and autophagosomes that play a key role in protein turnover and cellular homeostasis (Appelqvist et al., 2013). Substrates for degradation are delivered to lysosomes by two general routes namely endocytosis and autophagy. Endocytosis is responsible for up-taking extracellular nutrients as well as turnover of plasma membrane proteins. Autophagy, on the other hand, is responsible for removing intracellular protein aggregates and "worn out" organelles. Endolysosomes are especially important for physiological functions of neurons and skeletal muscle cells because they are long-lived post-mitotic cells that require efficient endolysosomes to degrade intracellular protein aggregates, eliminate "worn out" organelles and maintain membrane integrity. As such, endolysosome dysfunction in neurons has been linked to neurodegeneration and has been shown to play an early and important role in the development of AD (Cataldo et al., 2000; Nixon, 2005; Rajendran et al., 2008; Shimizu et al., 2008; Sannerud et al., 2011). Similarly, endolysosome dysfunction in skeletal muscle contributes to muscle degeneration and age-related disorders and has been implicated in a variety of autophagosome and lysosome related myopathies (Askanas et al., 2009; Nogalska et al., 2010; Malicdan and Nishino, 2012; Bonaldo and Sandri, 2013; Demontis et al., 2013; Sandri et al., 2013). Interestingly, skeletal muscle deficits such as loss of muscle mass and reduced muscle strength has been shown to be early signs of $\mathrm{AD}$ that contribute to disability and could predict the onset and progression of clinical AD (Buchman et al., 2007; Boyle et al., 2009; Burns et al., 2010). However, the question of whether endolysosome dysfunction leads to development of pathological features of $\mathrm{AD}$ in skeletal muscle and contributes to skeletal muscle deficits in $\mathrm{AD}$ has not been addressed. As such, we determined the extent to which elevated levels of plasma LDL cholesterol, a robust risk factor for $\mathrm{AD}$, disturbed the structure and function of endolysosomes and promoted the development of $\mathrm{AD}$-like pathological features including intracellular deposition of $A \beta / A \beta P P$, phosphorylated tau, and ubiquitin in skeletal muscle.

Given that plasma lipoproteins supply skeletal muscle with needed cholesterol (Spady and Dietschy, 1983), elevated plasma LDL cholesterol and subsequent increases in LDL cholesterol uptake could lead to increased cholesterol accumulation in endolysosomes and disturbed endolysosome structure and dysfunction. As expected, we demonstrated cholesterol-enriched diet increased free cholesterol in endosomes and lysosomes in skeletal muscle. Importantly, we demonstrated that cholesterolenriched diet dramatically enlarged endolysosomes and inhibited endolysosome function. Our observations that cholesterolenriched diet increased co-localization of EEA1 with LAMP2 and LC3 indicate that abnormally enlarged endolysosomes might result from uncontrolled fusion of early endosomes 
with late endosome/lysosomes or autophagosomes, a finding that is consistent with recent reports that EEA1 positive early endosomes could fuse with lysosomes under disrupted endocytic homeostasis (Falcon-Perez et al., 2005; Das and Pellett, 2011; Ramanathan et al., 2013).

The observations that cholesterol enriched diet increased accumulation of $\mathrm{A} \beta / \mathrm{A} \beta \mathrm{PP}$ in endolysosomes suggest to us that increased amyloidogenic processing of $\mathrm{A} \beta \mathrm{PP}$ was caused by morphological and functional alterations in endolysosomes. The observation that $A \beta$ is also present in LC3-positive autophagosomes suggests further the presence of dysfunctional endolysosomes because autophagic activity itself is part of the endolysosome system. Elevated levels of LDL cholesterol could promote $\mathrm{A} \beta$ deposition in skeletal muscle in two ways. First, receptor-mediated endocytosis of LDL cholesterol could encourage the accumulation of $\mathrm{A} \beta \mathrm{PP}$ in endolysosomes by either promoting $\mathrm{A} \beta \mathrm{PP}$ internalization (Waldron et al., 2006, 2008; Klug et al., 2011) or impairing the recycling of internalized A $\beta$ PP back to plasma membrane (Andersen et al., 2005; Willnow and Andersen, 2013). Second, endolysosome inhibition could lead to impairment of $A \beta$ degradation in lysosomes because $\mathrm{A} \beta$ can be degraded in lysosomes by cathepsin D and B (Nogalska et al., 2012; Saido and Leissring, 2012). Our observations that cholesterol-enriched diet increases endolysosome accumulation of $\mathrm{A} \beta \mathrm{PP}$ and decreased specific enzyme activities of cathepsin D and B support, at least indirectly, both possible mechanisms.

The observations that cholesterol-enriched diet increased accumulation of ubiquitin and phosphorylated tau in endolysosomes in skeletal muscle indicate that endolysosome dysfunction as induced by a cholesterol-enriched diet contributes to the development of AD-like ubiquitin-positive multi-protein aggregates and inclusions in skeletal muscle. These findings are consistent with other's reports that increased accumulation of cholesterol in lysosomes and subsequent lysosome dysfunction has been linked to the development of neurofibrillary tangles in brains of patients with Niemann-Pick type C disease (Sawamura et al., 2001; Bu et al., 2002; Distl et al., 2003; Vance, 2006; Bi and Liao, 2007; Liao et al., 2007) and our reports that

\section{REFERENCES}

Alonso, A. C., Grundke-Iqbal, I., and Iqbal, K. (1996). Alzheimer's disease hyperphosphorylated tau sequesters normal tau into tangles of filaments and disassembles microtubules. Nat. Med. 2, 783-787. doi: 10.1038/nm0796-783

Alonso Adel, C., ElAkkad, E., Gong, C., Liu, F., Tanaka, T., Kudo, T., et al. (2013). Inge Grundke-Iqbal, Ph.D. (1937-2012): the discoverer of the abnormal hyperphosphorylation of tau in Alzheimer's disease. J. Mol. Neurosci. 49, 430435. doi: 10.1007/s12031-012-9925-Z

Andersen, O. M., Reiche, J., Schmidt, V., Gotthardt, M., Spoelgen, R., Behlke, J., et al. (2005). Neuronal sorting protein-related receptor sorLA/LR11 regulates processing of the amyloid precursor protein. Proc. Natl. Acad. Sci. U.S.A. 102, 13461-13466. doi: 10.1073/pnas.0503689102

Annunziata, I., Patterson, A., Helton, D., Hu, H., Moshiach, S., Gomero, E., et al. (2013). Lysosomal NEU1 deficiency affects amyloid precursor protein levels and amyloid-beta secretion via deregulated lysosomal exocytosis. Nat. Commun. 4, 2734. doi: 10.1038/ncomms3734

Appelqvist, H., Waster, P., Kagedal, K., and Ollinger, K. (2013). The lysosome: from waste bag to potential therapeutic target. J. Mol. Cell Biol. 5, 214-226. doi: $10.1093 / \mathrm{jmcb} / \mathrm{mjt} 022$ endolysosome dysfunction as induced by high levels of LDL cholesterol contributes to the development of tau-pathology in neurons (Chen et al., 2010; Hui et al., 2012).

Although a causal relationship is not determined, our findings do suggest that endolysosome dysfunction as induced by elevated plasma LDL cholesterol, aging, or other factors may play a pathogenic role in the development of AD-like pathological changes observed in skeletal muscle, similar to its role in neurodegeneration and the pathogenesis of $\mathrm{AD}$ in brain. Furthermore, our findings support the notion that common pathogenic mechanisms may exist in skeletal muscle and neurons, and that the skeletal muscle changes may represent early and progressive pathological features of $\mathrm{AD}$.

\section{AUTHOR CONTRIBUTIONS}

$\mathrm{XC}, \mathrm{OG}$, and JG contributed to design the work. XC and JW contributed to the acquisition and analysis of data. $\mathrm{XC}$ drafted the work. XC, JW, OG, and JG contributed to interpretation of data and revising the work of intellectual content and final approval of the version to be published. XC drafted the work. XC, JW, OG, and JG approved of the final version and agreed to be accountable for all aspects of the work in ensuring that questions related to the accuracy or integrity of any part of the work are appropriately investigated and resolved.

\section{FUNDING}

This work was supported by P30GM103329, R01MH100972, and R01MH105329.

\section{SUPPLEMENTARY MATERIAL}

The Supplementary Material for this article can be found online at: http://journal.frontiersin.org/article/10.3389/fnagi. 2016.00129

Askanas, V., Engel, W. K., and Nogalska, A. (2009). Inclusion body myositis: a degenerative muscle disease associated with intra-muscle fiber multi-protein aggregates, proteasome inhibition, endoplasmic reticulum stress and decreased lysosomal degradation. Brain Pathol. 19, 493-506. doi: 10.1111/j.17503639.2009.00290.x

Bi, X., and Liao, G. (2007). Autophagic-lysosomal dysfunction and neurodegeneration in Niemann-Pick Type C mice: lipid starvation or indigestion? Autophagy 3, 646-648. doi: 10.4161/auto.5074

Bonaldo, P., and Sandri, M. (2013). Cellular and molecular mechanisms of muscle atrophy. Dis. Model Mech. 6, 25-39. doi: 10.1242/dmm.010389

Boyle, P. A., Buchman, A. S., Wilson, R. S., Leurgans, S. E., and Bennett, D. A. (2009). Association of muscle strength with the risk of Alzheimer disease and the rate of cognitive decline in community-dwelling older persons. Arch. Neurol. 66, 1339-1344. doi: 10.1001/archneurol.2009.240

Braak, H., and Del Tredici, K. (2004). Alzheimer's disease: intraneuronal alterations precede insoluble amyloid-beta formation. Neurobiol. Aging 25, 713-718; discussion 743-716. doi: 10.1016/j.neurobiolaging.2003.12.015

Brown, M. S., and Goldstein, J. L. (1986). A receptor-mediated pathway for cholesterol homeostasis. Science 232, 34-47. doi: 10.1126/science. 3513311 
Bu, B., Li, J., Davies, P., and Vincent, I. (2002). Deregulation of cdk5, hyperphosphorylation, and cytoskeletal pathology in the Niemann-Pick type C murine model. J. Neurosci. 22, 6515-6525.

Buchman, A. S., Boyle, P. A., Wilson, R. S., Tang, Y., and Bennett, D. A. (2007). Frailty is associated with incident Alzheimer's disease and cognitive decline in the elderly. Psychosom. Med. 69, 483-489. doi: 10.1097/psy.0b013e318068de1d

Burns, J. M., Johnson, D. K., Watts, A., Swerdlow, R. H., and Brooks, W. M. (2010). Reduced lean mass in early Alzheimer disease and its association with brain atrophy. Arch. Neurol. 67, 428-433. doi: 10.1001/archneurol.2010.38

Cataldo, A. M., Hamilton, D. J., and Nixon, R. A. (1994). Lysosomal abnormalities in degenerating neurons link neuronal compromise to senile plaque development in Alzheimer disease. Brain Res. 640, 68-80. doi: 10.1016/0006-8993(94)91858-9

Cataldo, A. M., and Nixon, R. A. (1990). Enzymatically active lysosomal proteases are associated with amyloid deposits in Alzheimer brain. Proc. Natl. Acad. Sci. U.S.A. 87, 3861-3865. doi: 10.1073/pnas.87.10.3861

Cataldo, A. M., Peterhoff, C. M., Troncoso, J. C., Gomez-Isla, T., Hyman, B. T., and Nixon, R. A. (2000). Endocytic pathway abnormalities precede amyloid beta deposition in sporadic Alzheimer's disease and Down syndrome: differential effects of APOE genotype and presenilin mutations. Am. J. Pathol. 157, 277-286. doi: $10.1016 /$ S0002-9440(10)64538-5

Chen, X., Gawryluk, J. W., Wagener, J. F., Ghribi, O., and Geiger, J. D. (2008a). Caffeine blocks disruption of blood brain barrier in a rabbit model of Alzheimer's disease. J. Neuroinflammation 5, 12. doi: 10.1186/1742-2094-5-12

Chen, X., Ghribi, O., and Geiger, J. D. (2008b). Rabbits fed cholesterolenriched diets exhibit pathological features of inclusion body myositis. Am. J. Physiol. Regul. Integr. Comp. Physiol. 294, R829-R835. doi: 10.1152/ajpregu. 00639.2007

Chen, X., Wagener, J. F., Morgan, D. H., Hui, L., Ghribi, O., and Geiger, J. D. (2010). Endolysosome mechanisms associated with Alzheimer's disease-like pathology in rabbits ingesting cholesterol-enriched diet. J. Alzheimers Dis. 22, 1289-1303. doi: 10.3233/JAD-2010-101323

Chesser, A. S., Pritchard, S. M., and Johnson, G. V. (2013). Tau clearance mechanisms and their possible role in the pathogenesis of Alzheimer disease. Front. Neurol. 4:122. doi: 10.3389/fneur.2013.00122

D'Agostino, C., Nogalska, A., Cacciottolo, M., Engel, W. K., and Askanas, V. (2011). Abnormalities of NBR1, a novel autophagy-associated protein, in muscle fibers of sporadic inclusion-body myositis. Acta Neuropathol. 122, 627-636. doi: 10.1007/s00401-011-0874-3

Das, S., and Pellett, P. E. (2011). Spatial relationships between markers for secretory and endosomal machinery in human cytomegalovirus-infected cells versus those in uninfected cells. J. Virol. 85, 5864-5879. doi: 10.1128/JVI.00155-11

Demontis, F., Piccirillo, R., Goldberg, A. L., and Perrimon, N. (2013). Mechanisms of skeletal muscle aging: insights from Drosophila and mammalian models. Dis. Model Mech. 6, 1339-1352. doi: 10.1242/dmm.012559

Dil Kuazi, A., Kito, K., Abe, Y., Shin, R. W., Kamitani, T., and Ueda, N. (2003). NEDD8 protein is involved in ubiquitinated inclusion bodies. J. Pathol. 199, 259-266. doi: 10.1002/path.1283

Distl, R., Treiber-Held, S., Albert, F., Meske, V., Harzer, K., and Ohm, T. G. (2003). Cholesterol storage and tau pathology in Niemann-Pick type C disease in the brain. J. Pathol. 200, 104-111. doi: 10.1002/path.1320

Falcon-Perez, J. M., Nazarian, R., Sabatti, C., and Dell'Angelica, E. C. (2005). Distribution and dynamics of Lamp1-containing endocytic organelles in fibroblasts deficient in BLOC-3. J. Cell Sci. 118, 5243-5255. doi: $10.1242 /$ jcs. 02633

Ghribi, O. (2006). Preservation of the blood brain barrier integrity may underlie neuroprotective effects of statins in Alzheimer's disease. J. Alzheimers Dis. 10, 407-408.

Ghribi, O., Larsen, B., Schrag, M., and Herman, M. M. (2006). High cholesterol content in neurons increases BACE, beta-amyloid, and phosphorylated tau levels in rabbit hippocampus. Exp. Neurol. 200, 460-467. doi: 10.1016/j.expneurol.2006.03.019

Goate, A., and Hardy, J. (2012). Twenty years of Alzheimer's diseasecausing mutations. J. Neurochem. 120(Suppl. 1), 3-8. doi: 10.1111/j.14714159.2011.07575.x

Grbovic, O. M., Mathews, P. M., Jiang, Y., Schmidt, S. D., Dinakar, R., Summers-Terio, N. B., et al. (2003). Rab5-stimulated up-regulation of the endocytic pathway increases intracellular beta-cleaved amyloid precursor protein carboxyl-terminal fragment levels and Abeta production. J. Biol. Chem. 278, 31261-31268. doi: 10.1074/jbc.M304122200

Hamano, T., Gendron, T. F., Causevic, E., Yen, S. H., Lin, W. L., Isidoro, C., et al. (2008). Autophagic-lysosomal perturbation enhances tau aggregation in transfectants with induced wild-type tau expression. Eur. J. Neurosci. 27, 1119-1130. doi: 10.1111/j.1460-9568.2008.06084.x

Hebert, L. E., Weuve, J., Scherr, P. A., and Evans, D. A. (2013). Alzheimer disease in the United States (2010-2050) estimated using the 2010 census. Neurology 80 , 1778-1783. doi: 10.1212/WNL.0b013e31828726f5

Holler, D., and Dikic, I. (2004). Receptor endocytosis via ubiquitin-dependent and -independent pathways. Biochem. Pharmacol. 67, 1013-1017. doi: 10.1016/j.bcp.2004.01.003

Holtzman, D. M., Morris, J. C., and Goate, A. M. (2011). Alzheimer's disease: the challenge of the second century. Sci. Transl. Med. 3:77sr71. doi: 10.1126/scitranslmed.3002369

Hui, L., Chen, X., and Geiger, J. D. (2012). Endolysosome involvement in LDL cholesterol-induced Alzheimer's disease-like pathology in primary cultured neurons. Life Sci. 91, 1159-1168. doi: 10.1016/j.lfs.2012.04.039

Jiang, S., Li, Y., Zhang, X., Bu, G., Xu, H., and Zhang, Y. W. (2014). Trafficking regulation of proteins in Alzheimer's disease. Mol. Neurodegener. 9, 6. doi: $10.1186 / 1750-1326-9-6$

Kenessey, A., Nacharaju, P., Ko, L. W., and Yen, S. H. (1997). Degradation of tau by lysosomal enzyme cathepsin D: implication for Alzheimer neurofibrillary degeneration. J. Neurochem. 69, 2026-2038. doi: 10.1046/j.14714159.1997.69052026.x

Klug, W., Dietl, A., Simon, B., Sinning, I., and Wild, K. (2011). Phosphorylation of LRP1 regulates the interaction with Fe65. FEBS Lett. 585, 3229-3235. doi: 10.1016/j.febslet.2011.09.028

Kuo, Y. M., Kokjohn, T. A., Watson, M. D., Woods, A. S., Cotter, R. J., Sue, L. I., et al. (2000). Elevated abeta 42 in skeletal muscle of Alzheimer disease patients suggests peripheral alterations of AbetaPP metabolism. Am. J. Pathol. 156, 797-805. doi: 10.1016/S0002-9440(10)64947-4

LaFerla, F. M., Green, K. N., and Oddo, S. (2007). Intracellular amyloid-beta in Alzheimer's disease. Nat. Rev. Neurosci. 8, 499-509. doi: 10.1038/nrn2168

Lesser, G. T., Beeri, M. S., Schmeidler, J., Purohit, D. P., and Haroutunian, V. (2011). Cholesterol and LDL relate to neuritic plaques and to APOE4 presence but not to neurofibrillary tangles. Curr. Alzheimer Res. 8, 303-312. doi: $10.2174 / 156720511795563755$

Liao, G., Yao, Y., Liu, J., Yu, Z., Cheung, S., Xie, A., et al. (2007). Cholesterol accumulation is associated with lysosomal dysfunction and autophagic stress in Npc1 -/- mouse brain. Am. J. Pathol. 171, 962-975. doi: 10.2353/ajpath.2007.070052

Ma, Q. L., Galasko, D. R., Ringman, J. M., Vinters, H. V., Edland, S. D., Pomakian, J., et al. (2009). Reduction of SorLA/LR11, a sorting protein limiting beta-amyloid production, in Alzheimer disease cerebrospinal fluid. Arch. Neurol. 66, 448-457. doi: 10.1001/archneurol.2009.22

Malicdan, M. C., and Nishino, I. (2012). Autophagy in lysosomal myopathies. Brain Pathol. 22, 82-88. doi: 10.1111/j.1750-3639.2011.00543.x

Miners, J. S., Barua, N., Kehoe, P. G., Gill, S., and Love, S. (2011). Abeta-degrading enzymes: potential for treatment of Alzheimer disease. J. Neuropathol. Exp. Neurol. 70, 944-959. doi: 10.1097/NEN.0b013e3182345e46

Morel, E., Chamoun, Z., Lasiecka, Z. M., Chan, R. B., Williamson, R. L., Vetanovetz, C., et al. (2013). Phosphatidylinositol-3-phosphate regulates sorting and processing of amyloid precursor protein through the endosomal system. Nat. Commun. 4, 2250. doi: 10.1038/ncomms3250

Nilsson, P., Loganathan, K., Sekiguchi, M., Matsuba, Y., Hui, K., Tsubuki, S., et al. (2013). Abeta secretion and plaque formation depend on autophagy. Cell Rep. 5, 61-69. doi: 10.1016/j.celrep.2013.08.042

Nixon, R. A. (2005). Endosome function and dysfunction in Alzheimer's disease and other neurodegenerative diseases. Neurobiol. Aging 26, 373-382. doi: 10.1016/j.neurobiolaging.2004.09.018

Nixon, R. A. (2007). Autophagy, amyloidogenesis and Alzheimer disease. J. Cell Sci. 120, 4081-4091. doi: 10.1242/jcs. 019265

Nogalska, A., D’Agostino, C., Engel, W. K., and Askanas, V. (2012). Activation of the gamma-secretase complex and presence of gamma-secretaseactivating protein may contribute to Abeta 42 production in sporadic inclusion-body myositis muscle fibers. Neurobiol. Dis. 48, 141-149. doi: 10.1016/j.nbd.2012.06.008 
Nogalska, A., D’Agostino, C., Terracciano, C., Engel, W. K., and Askanas, V. (2010). Impaired autophagy in sporadic inclusion-body myositis and in endoplasmic reticulum stress-provoked cultured human muscle fibers. Am. J. Pathol. 177, 1377-1387. doi: 10.2353/ajpath.2010.100050

Oyama, F., Murakami, N., and Ihara, Y. (1998). Chloroquine myopathy suggests that tau is degraded in lysosomes: implication for the formation of paired helical filaments in Alzheimer's disease. Neurosci. Res. 31, 1-8. doi: 10.1016/S01680102(98)00020-0

Rajendran, L., and Annaert, W. (2012). Membrane trafficking pathways in Alzheimer's disease. Traffic 13, 759-770. doi: 10.1111/j.1600-0854.2012. 01332.x

Rajendran, L., Schneider, A., Schlechtingen, G., Weidlich, S., Ries, J., Braxmeier, T., et al. (2008). Efficient inhibition of the Alzheimer's disease beta-secretase by membrane targeting. Science 320, 520-523. doi: 10.1126/science.1156609

Ramanathan, H. N., Zhang, G., and Ye, Y. (2013). Monoubiquitination of EEA1 regulates endosome fusion and trafficking. Cell Biosci. 3, 24. doi: 10.1186/20453701-3-24

Reed, B., Villeneuve, S., Mack, W., DeCarli, C., Chui, H. C., and Jagust, W. (2014). Associations between serum cholesterol levels and cerebral amyloidosis. JAMA Neurol. 71, 195-200. doi: 10.1001/jamaneurol.2013.5390

Saido, T., and Leissring, M. A. (2012). Proteolytic degradation of amyloid beta-protein. Cold Spring Harb. Perspect. Med. 2:a006379. doi: 10.1101/cshperspect.a006379

Sandri, M., Coletto, L., Grumati, P., and Bonaldo, P. (2013). Misregulation of autophagy and protein degradation systems in myopathies and muscular dystrophies. J. Cell Sci. 126, 5325-5333. doi: 10.1242/jcs.114041

Sannerud, R., Declerck, I., Peric, A., Raemaekers, T., Menendez, G., Zhou, L., et al. (2011). ADP ribosylation factor 6 (ARF6) controls amyloid precursor protein (APP) processing by mediating the endosomal sorting of BACE1. Proc. Natl. Acad. Sci. U.S.A. 108, E559-E568. doi: 10.1073/pnas. 1100745108

Sawamura, N., Gong, J. S., Garver, W. S., Heidenreich, R. A., Ninomiya, H., Ohno, K., et al. (2001). Site-specific phosphorylation of tau accompanied by activation of mitogen-activated protein kinase (MAPK) in brains of Niemann-Pick type C mice. J. Biol. Chem. 276, 10314-10319. doi: 10.1074/jbc.M009733200

Shimizu, H., Tosaki, A., Kaneko, K., Hisano, T., Sakurai, T., and Nukina, N. (2008). Crystal structure of an active form of BACE1, an enzyme responsible for amyloid beta protein production. Mol. Cell. Biol. 28, 3663-3671. doi: 10.1128/MCB.02185-07

Solomon, A., Kivipelto, M., Wolozin, B., Zhou, J., and Whitmer, R. A. (2009). Midlife serum cholesterol and increased risk of Alzheimer's and vascular dementia three decades later. Dement. Geriatr. Cogn. Disord. 28, 75-80. doi: $10.1159 / 000231980$

Spady, D. K., and Dietschy, J. M. (1983). Sterol synthesis in vivo in 18 tissues of the squirrel monkey, guinea pig, rabbit, hamster, and rat. J. Lipid Res. 24, 303-315.

Sparks, D. L., Scheff, S. W., Hunsaker, J. C. III, Liu, H., Landers, T., and Gross, D. R. (1994). Induction of Alzheimer-like beta-amyloid immunoreactivity in the brains of rabbits with dietary cholesterol. Exp. Neurol. 126, 88-94. doi: 10.1006/exnr.1994.1044

Tooze, S. A., and Razi, M. (2009). The essential role of early endosomes in autophagy is revealed by loss of COPI function. Autophagy 5, 874-875. doi: 10.4161/auto.9066

Torres, M., Jimenez, S., Sanchez-Varo, R., Navarro, V., Trujillo-Estrada, L., Sanchez-Mejias, E., et al. (2012). Defective lysosomal proteolysis and axonal transport are early pathogenic events that worsen with age leading to increased APP metabolism and synaptic Abeta in transgenic APP/PS1 hippocampus. Mol. Neurodegener. 7, 59. doi: 10.1186/1750-1326-7-59

Vance, J. E. (2006). Lipid imbalance in the neurological disorder, Niemann-Pick C disease. FEBS Lett. 580, 5518-5524. doi: 10.1016/j.febslet.2006.06.008

Waldron, E., Heilig, C., Schweitzer, A., Nadella, N., Jaeger, S., Martin, A. M., et al. (2008). LRP1 modulates APP trafficking along early compartments of the secretory pathway. Neurobiol. Dis. 31, 188-197. doi: 10.1016/j.nbd.2008.04.006

Waldron, E., Jaeger, S., and Pietrzik, C. U. (2006). Functional role of the low-density lipoprotein receptor-related protein in Alzheimer's disease. Neurodegener. Dis. 3, 233-238. doi: 10.1159/000095261

Wang, J. Z., Xia, Y. Y., Grundke-Iqbal, I., and Iqbal, K. (2013). Abnormal hyperphosphorylation of tau: sites, regulation, and molecular mechanism of neurofibrillary degeneration. J. Alzheimers Dis. 33(Suppl. 1), S123-S139. doi: 10.3233/JAD-2012-129031

Wang, Y., Martinez-Vicente, M., Kruger, U., Kaushik, S., Wong, E., Mandelkow, E. M., et al. (2009). Tau fragmentation, aggregation and clearance: the dual role of lysosomal processing. Hum. Mol. Genet. 18, 4153-4170. doi: 10.1093/hmg/ddp367

Willnow, T. E., and Andersen, O. M. (2013). Sorting receptor SORLA-a trafficking path to avoid Alzheimer disease. J. Cell Sci. 126, 2751-2760. doi: $10.1242 /$ jcs. 125393

Wolfe, D. M., Lee, J. H., Kumar, A., Lee, S., Orenstein, S. J., and Nixon, R. A. (2013). Autophagy failure in Alzheimer's disease and the role of defective lysosomal acidification. Eur. J. Neurosci. 37, 1949-1961. doi: 10.1111/ejn.12169

Zou, C., Montagna, E., Shi, Y., Peters, F., Blazquez-Llorca, L., Shi, S., et al. (2015). Intraneuronal APP and extracellular Abeta independently cause dendritic spine pathology in transgenic mouse models of Alzheimer's disease. Acta Neuropathol. 129, 909-920. doi: 10.1007/s00401-015-1421-4

Conflict of Interest Statement: The authors declare that the research was conducted in the absence of any commercial or financial relationships that could be construed as a potential conflict of interest.

Copyright (c) 2016 Chen, Wagener, Ghribi and Geiger. This is an open-access article distributed under the terms of the Creative Commons Attribution License (CC BY). The use, distribution or reproduction in other forums is permitted, provided the original author(s) or licensor are credited and that the original publication in this journal is cited, in accordance with accepted academic practice. No use, distribution or reproduction is permitted which does not comply with these terms. 